\title{
Pacific
}

Journal of

Mathematics

\section{RICCI TENSOR OF REAL HYPERSURFACES}

MAYUKO KON 


\title{
RICCI TENSOR OF REAL HYPERSURFACES
}

\author{
MAYUKO KON
}

\begin{abstract}
Let $M$ be a real hypersurface of a complex space form $M^{n}(c), c \neq 0$, and suppose that the structure vector field $\xi$ is an eigen vector field of the Ricci tensor $S$, which satisfies $S \xi=\beta \xi$ where $\beta$ is a function. We show that if $\left(\nabla_{X} S\right) Y$ is proportional to $\xi$ for any vector fields $X$ and $Y$ orthogonal to $\xi$, then $M$ is a Hopf hypersurface, and if it is perpendicular to $\xi$, then $M$ is a ruled real hypersurface.
\end{abstract}

\section{Introduction}

Takagi [1973] gave a classification of the homogeneous real hypersurface (see also [Takagi 1975a; 1975b]). As a consequence of this result, the structure vector $\xi$ of any homogeneous real hypersurface in $\mathbb{C} P^{n}$ is principal. If $\xi$ satisfies this property, then $M$ is said to be a Hopf hypersurface. When the ambient manifold is a complex hyperbolic space, Lohnherr [1998] (see also [Lohnherr and Reckziegel 1999]) discovered a homogeneous ruled real hypersurface in $\mathbb{C} H^{n}$ that is not a Hopf hypersurface, and further examples were given (see [Berndt and Brück 2001]). The classification theorem for homogeneous real hypersurfaces in $\mathbb{C} H^{n}, n \geq 2$, was given by Berndt and Tamaru [2007].

When a real hypersurface is Hopf, fundamental formulas are simple. So many classification theorems are given under that assumption (see, for example, [Niebergall and Ryan 1997]). Kimura [1986] has given a classification of Hopf hypersurfaces of $\mathbb{C} P^{n}, n \geq 2$, with constant principal curvatures. He showed that a real hypersurface in $\mathbb{C} P^{n}$ with constant principal curvatures is a Hopf hypersurface if and only if it is an open part of a homogeneous real hypersurface. A classification theorem for Hopf hypersurfaces with constant principal curvatures in $\mathbb{C} H^{n}, n \geq 2$, was given by Berndt [1989].

On the other hand, the Ricci tensor of the real hypersurfaces is an interesting subject. It is well known that any real hypersurface of $M^{n}(c), c \neq 0$, is not Einstein. If the Ricci tensor $S$ is of the form $S(X, Y)=a g(X, Y)+b \eta(X) \eta(Y)$, then the real hypersurface is said to be pseudo-Einstein. The classification theorems for pseudo-Einstein real hypersurfaces in a complex space form $M^{n}(c)$ have been

MSC2010: primary 53C40; secondary 53C55, 53C25.

Keywords: real hypersurface, Ricci tensor. 
completed [Cecil and Ryan 1982; Kim and Ryan 2008; Kon 1979; Montiel 1985]. $\mathrm{Ki}$ [1989] showed that there are no real hypersurfaces with parallel Ricci tensor, $\nabla S=0$, in $M^{n}(c), n \geq 3$. Several conditions that weaken the condition $\nabla S=0$ have been studied (see [Ki et al. 1990; Suh 1990]).

We focus on the Ricci tensor $S$ and consider a condition $S \xi=\beta \xi$, where $\beta$ is a function. We note that this condition contains not only Hopf hypersurfaces, $A \xi=\alpha \xi$, but also some non-Hopf hypersurfaces. For example, ruled hypersurfaces, which are an important example of non-Hopf hypersurfaces, also satisfy $S \xi=\beta \xi$. Under this assumption, we study some Hopf hypersurfaces and ruled real hypersurfaces according to the direction of a covariant differentiation of $S$.

Our main result is the following theorem:

Theorem 1.1. Let $M$ be a connected real hypersurface of $M^{n}(c), c \neq 0$, and suppose that the Ricci tensor $S$ of $M$ satisfies $S \xi=\beta \xi$ for some function $\beta$.

(1) If $\left(\nabla_{X} S\right) Y$ is proportional to the structure vector field $\xi$ for any vector fields $X$ and $Y$ orthogonal to $\xi$, then $M$ is a Hopf hypersurface.

(2) If $\left(\nabla_{X} S\right) Y$ is perpendicular to the structure vector field $\xi$ for any vector fields $X$ and $Y$ orthogonal to the structure vector field $\xi$, then $M$ is a ruled real hypersurface.

When $n=2$, the author gave a corresponding result in [Kon 2014].

\section{Preliminaries}

Let $M^{n}(c)$ denote the complex space form of complex dimension $n$ (real dimension $2 n$ ) with constant holomorphic sectional curvature $4 c$. We denote by $J$ the almost complex structure of $M^{n}(c)$. The Hermitian metric of $M^{n}(c)$ is denoted by $G$.

Let $M$ be a real $(2 n-1)$-dimensional hypersurface immersed in $M^{n}(c)$. Throughout this paper, we suppose that $M$ is connected. We denote by $g$ the Riemannian metric induced on $M$ from $G$. We take the unit normal vector field $N$ of $M$ in $M^{n}(c)$. For any vector field $X$ tangent to $M$, we define $\phi, \eta$ and $\xi$ by

$$
J X=\phi X+\eta(X) N, \quad J N=-\xi,
$$

where $\phi X$ is the tangential part of $J X, \phi$ is a tensor field of type $(1,1), \eta$ is a 1 -form, and $\xi$ is the unit vector field on $M$. We call $\xi$ the structure vector field. Then

$$
\phi^{2} X=-X+\eta(X) \xi, \quad \phi \xi=0, \quad \eta(\phi X)=0
$$

for any vector field $X$ tangent to $M$. Moreover, we have $g(\phi X, Y)+g(X, \phi Y)=0, \quad \eta(X)=g(X, \xi), \quad g(\phi X, \phi Y)=g(X, Y)-\eta(X) \eta(Y)$.

Thus $(\phi, \xi, \eta, g)$ defines an almost contact metric structure on $M$. 
We denote by $\tilde{\nabla}$ the operator of covariant differentiation in $M^{n}(c)$, and by $\nabla$ the operator of covariant differentiation in $M$ determined by the induced metric. Then the Gauss and Weingarten formulas are given respectively by

$$
\tilde{\nabla}_{X} Y=\nabla_{X} Y+g(A X, Y) N, \quad \tilde{\nabla}_{X} N=-A X,
$$

for any vector fields $X$ and $Y$ tangent to $M$.

For the contact metric structure on $M$, we have

$$
\nabla_{X} \xi=\phi A X, \quad\left(\nabla_{X} \phi\right) Y=\eta(Y) A X-g(A X, Y) \xi .
$$

We call $A$ the shape operator of $M$. If the shape operator $A$ of $M$ satisfies $A \xi=\alpha \xi$ for some function $\alpha$, then $M$ is called a Hopf hypersurface. By the Codazzi equation, we have the following result (see [Maeda 1976]).

Proposition A. Let $M$ be a Hopf hypersurface in $M^{n}(c), n \geq 2$. If $X \perp \xi$ and $A X=\lambda X$, then $\alpha=g(A \xi$, $\xi)$ is constant and

$$
(2 \lambda-\alpha) A \phi X=(\lambda \alpha+2 c) \phi X .
$$

We offer an important example of a non-Hopf hypersurface. Take a regular curve $\gamma$ in $M^{n}(c)$ with tangent vector field $X$. At each point of $\gamma$ there is a unique complex projective or hyperbolic hyperplane cutting $\gamma$ so as to be orthogonal to $X$ and $J X$. The union of these hyperplanes is called a ruled real hypersurface (see [Kimura and Maeda 1989; Lohnherr and Reckziegel 1999; Niebergall and Ryan 1997]).

We remark that the shape operator $A$ is $\eta$-parallel if it satisfies $g\left(\left(\nabla_{X} A\right) Y, Z\right)=0$ for any $X, Y$ and $Z$ orthogonal to $\xi$.

We denote by $R$ the Riemannian curvature tensor field of $M$. Then the equation of Gauss is given by

$R(X, Y) Z$

$$
\begin{aligned}
=c\{ & g(Y, Z) X-g(X, Z) Y+g(\phi Y, Z) \phi X-g(\phi X, Z) \phi Y-2 g(\phi X, Y) \phi Z\} \\
& +g(A Y, Z) A X-g(A X, Z) A Y,
\end{aligned}
$$

and the equation of Codazzi by

$$
\left(\nabla_{X} A\right) Y-\left(\nabla_{Y} A\right) X=c\{\eta(X) \phi Y-\eta(Y) \phi X-2 g(\phi X, Y) \xi\} .
$$

From the equation of Gauss, the Ricci tensor $S$ of $M$ is given by

(1) $g(S X, Y)=(2 n+1) c g(X, Y)-3 c \eta(X) \eta(Y)+\operatorname{tr} A g(A X, Y)-g(A X, A Y)$,

where $\operatorname{tr} A$ is the trace of $A$. Taking a covariant differentiation, we have

(2) $g\left(\left(\nabla_{X} S\right) Y, Z\right)=-3 \operatorname{cg}(Y, \phi A X) \eta(Z)-3 c g(\phi A X, Z) \eta(Y)+(X \operatorname{tr} A) g(A Y, Z)$

$$
+\operatorname{tr} A g\left(\left(\nabla_{X} A\right) Y, Z\right)-g\left(\left(\nabla_{X} A\right) A Y, Z\right)-g\left(\left(\nabla_{X} A\right) Y, A Z\right) \text {. }
$$


Now we develop some lemmas needed to prove our main theorem. Suppose $n \geq 3$.

Lemma 2.1. Let $M$ be a real hypersurface in a complex space form $M^{n}(c), n \geq 3$, $c \neq 0$. If there exists an orthonormal frame $\left\{\xi, e_{1}, \ldots, e_{2 n-2}\right\}$ on a sufficiently small neighborhood $\mathcal{N}$ of $x \in M$ such that the shape operator $A$ can be represented as

then we have

$$
A=\left(\begin{array}{ccccc}
\alpha & h_{1} & 0 & \cdots & 0 \\
h_{1} & a_{1} & & & \\
0 & & a_{2} & & \vdots \\
\vdots & & & \ddots & 0 \\
0 & & \cdots & 0 & a_{2 n-2}
\end{array}\right),
$$

(4) $\left(a_{j}-a_{1}\right) g\left(\nabla_{e_{i}} e_{j}, e_{1}\right)-\left(a_{i}-a_{1}\right) g\left(\nabla_{e_{j}} e_{i}, e_{1}\right)=h_{1}\left(a_{i}+a_{j}\right) g\left(e_{i}, \phi e_{j}\right)$,

(5) $h_{1} g\left(\nabla_{e_{i}} e_{j}, e_{1}\right)-h_{1} g\left(\nabla_{e_{j}} e_{i}, e_{1}\right)=\left\{2 c-2 a_{i} a_{j}+\alpha\left(a_{i}+a_{j}\right)\right\} g\left(\phi e_{i}, e_{j}\right)$,

(17) $\left(a_{1}-a_{i}\right) g\left(\nabla_{\xi} e_{1}, e_{i}\right)-h_{1} g\left(\nabla_{e_{1}} e_{1}, e_{i}\right)=\left(c+a_{1} \alpha-a_{1} a_{i}-h_{1}^{2}\right) g\left(e_{i}, \phi e_{1}\right)$, for any $i, j \geq 2, i \neq j$.

Proof. By the equation of Codazzi, we have

$$
g\left(\left(\nabla_{e_{i}} A\right) e_{1}-\left(\nabla_{e_{1}} A\right) e_{i}, e_{j}\right)=0,
$$

where $i, j=2, \ldots, 2 n-2$. On the other hand, we have

$$
\begin{aligned}
g\left(\left(\nabla_{e_{i}} A\right) e_{1}-\left(\nabla_{e_{1}} A\right) e_{i}, e_{j}\right) & \\
& =g\left(\nabla_{e_{i}}\left(A e_{1}\right)-A \nabla_{e_{i}} e_{1}-\nabla_{e_{1}}\left(A e_{i}\right)+A \nabla_{e_{1}} e_{i}, e_{j}\right) \\
& =\left(a_{1}-a_{j}\right) g\left(\nabla_{e_{i}} e_{1}, e_{j}\right)+\left(a_{j}-a_{i}\right) g\left(\nabla_{e_{1}} e_{i}, e_{j}\right)+a_{i} h_{1} g\left(\phi e_{i}, e_{j}\right) .
\end{aligned}
$$


Thus we obtain (8). We obtain the other results through similar computations.

We remark that these equations hold in the case that $M$ is a Hopf hypersurface, i.e., $h_{1}=0$. When $n=2$, we showed the corresponding result in [Kon 2014].

We define the subspace $L_{x} \subset T_{x}(M)$ as the smallest subspace that contains $\xi$ and is invariant under the shape operator $A$. Then $M$ is Hopf if and only if $L_{x}$ is one-dimensional at each point $x$.

Lemma 2.2. Let $M$ be a real hypersurface of $M^{n}(c)$. If the Ricci tensor $S$ of $M$ satisfies $S \xi=\beta \xi$ for some function $\beta$, then $\operatorname{dim} L_{x} \leq 2$ at each point $x$ of $M^{n}(c)$.

Proof. By (1), we have

$$
0=g(S \xi, Y)=-g\left(A^{2} \xi, Y\right)
$$

for any $Y$ orthogonal to $\xi$ and $A \xi$. So $A^{2} \xi$ is spanned by $\xi$ and $A \xi$. Thus we see that $\operatorname{dim} L_{x} \leq 2$.

Suppose that $M$ is not a Hopf hypersurface and that $S \xi=\beta \xi$. By Lemma 2.2, we can take an orthonormal frame $\left\{\xi, e_{1}, \ldots, e_{2 n-2}\right\}$, locally, such that $A$ is of the form

$$
A=\left(\begin{array}{ccccc}
\alpha & h_{1} & & & 0 \\
h_{1} & a_{1} & & & \\
& & a_{2} & & \\
& & & \ddots & \\
0 & & & & a_{2 n-2}
\end{array}\right),
$$

where $h_{1}=g\left(A e_{1}, \xi\right), a_{i}=g\left(A e_{i}, e_{i}\right)$ for $i=1, \ldots, 2 n-2, g\left(A e_{i}, e_{j}\right)=0$ for $i \neq j$ and $\alpha=g(A \xi, \xi)$. By (1), we obtain

$$
\begin{aligned}
S \xi & =(2 n-2) c \xi+(\operatorname{tr} A)\left(h_{1} e_{1}+\alpha \xi\right)-A\left(h_{1} e_{1}+\alpha \xi\right) \\
& =\left(\operatorname{tr} A-\alpha-a_{1}\right) h_{1} e_{1}+\left\{(2 n-2) c+(\operatorname{tr} A) \alpha-h_{1}^{2}-\alpha^{2}\right\} \xi=\beta \xi .
\end{aligned}
$$

So we see that

$$
\operatorname{tr} A=\alpha+a_{1}, \quad a_{2}+\cdots+a_{2 n-2}=0 .
$$

Moreover, (1) implies that the Ricci tensor $S$ can be represented as

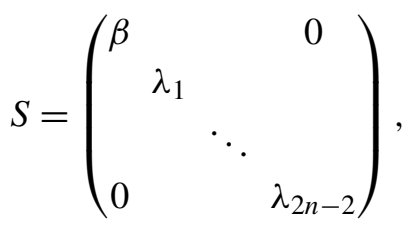

where $\beta$ and $\lambda_{i}$ satisfy

$$
\begin{gathered}
\beta=(2 n-2) c+\left(\alpha a_{1}-h_{1}^{2}\right), \quad \lambda_{1}=(2 n+1) c+\left(\alpha a_{1}-h_{1}^{2}\right), \\
\lambda_{j}=(2 n+1) c+\operatorname{tr} A \cdot a_{j}-a_{j}^{2}, \quad j=2, \ldots, 2 n-2 .
\end{gathered}
$$




\section{Real hypersurfaces with $\eta$-parallel Ricci tensor}

In this section, we consider the additional condition that the Ricci operator $S$ is $\eta$-parallel, that is,

$$
g\left(\left(\nabla_{X} S\right) Y, Z\right)=0
$$

for any vector fields $X, Y$ and $Z$ orthogonal to $\xi$. This is equivalent to the condition that $\left(\nabla_{X} S\right) Y$ is proportional to $\xi$ [Suh 1990].

Theorem 3.1. Let $M$ be a real hypersurface of $M^{n}(c), c \neq 0$, with $\eta$-parallel Ricci tensor. If the Ricci tensor $S$ of $M$ satisfies $S \xi=\beta \xi$ for some function $\beta$, then $M$ is a Hopf hypersurface.

Before proving Theorem 3.1, we need the following lemma.

Lemma 3.2. Let $M$ be a real hypersurface of $M^{n}(c), c \neq 0$, with $\eta$-parallel Ricci tensor. If the Ricci tensor $S$ of $M$ satisfies $S \xi=\beta \xi$ for some function $\beta$, then we have

$$
\begin{aligned}
g((R(W, X) S) Y, Z)=- & g(S \phi A X, Z) g(\phi A W, Y)-g(S \phi A X, Y) g(\phi A W, Z) \\
& +g(S \phi A W, Z) g(\phi A X, Y)+g(S \phi A W, Y) g(\phi A X, Z) \\
& -g\left(\left(\nabla_{\xi} S\right) Y, Z\right) g((\phi A+A \phi) X, W)
\end{aligned}
$$

for any $X, Y, Z$ and $W$ orthogonal to $\xi$.

Proof. Since $S$ is $\eta$-parallel, we have

$$
\begin{aligned}
& g((R(W, X) S) Y, Z) g( \\
&=g(W, X) S Y, Z)-g(R(W, X) Y, S Z) \\
&=g\left(\nabla_{W} \nabla_{X} S Y-\nabla_{X} \nabla_{W} S Y-\nabla_{[W, X]} S Y, Z\right) \\
& \quad-g\left(\nabla_{W} \nabla_{X} Y-\nabla_{X} \nabla_{W} Y-\nabla_{[W, X]} Y, S Z\right) \\
&=- g\left(\left(\nabla_{X} S\right) Y, \nabla_{W} Z\right)+g\left(\nabla_{W}\left(S \nabla_{X} Y\right), Z\right)+g\left(\left(\nabla_{W} S\right) Y, \nabla_{X} Z\right) \\
&-g\left(\nabla_{X}\left(S \nabla_{W} Y\right), Z\right)-g\left(\left(\nabla_{[W, X]} S\right) Y, Z\right)-g\left(\nabla_{W} \nabla_{Y}, S Z\right) \\
&+g\left(\nabla_{X} \nabla_{W} Y, S Z\right) \\
&=- g\left(\left(\nabla_{X} S\right) Y, \xi\right) g\left(\xi, \nabla_{W} Z\right)+g\left(\left(\nabla_{W} S\right) \nabla_{X} Y, Z\right) \\
&+g\left(\left(\nabla_{W} S\right) Y, \xi\right) g\left(\xi, \nabla_{X} Z\right)-g\left(\left(\nabla_{X} S\right) \nabla_{W} Y, Z\right) \\
&-g\left(\left(\nabla_{\xi} S\right) Y, Z\right) g(\xi,[W, X]) \\
&=- g(S \phi A X, Y) g(\phi A W, Z)+g(S \phi A W, Z) g(\phi A X, Y) \\
&+g(S \phi A W, Y) g(\phi A X, Z)-g(S \phi A X, Z) g(\phi A W, Y) \\
&-g\left(\left(\nabla_{\xi} S\right) Y, Z\right) g((\phi A+A \phi) X, W) .
\end{aligned}
$$

From Lemma 3.2 we obtain the following: 
Lemma 3.3. Let $M$ be a real hypersurface of $M^{n}(c), c \neq 0$, with $\eta$-parallel Ricci tensor. Suppose that the Ricci tensor $S$ of $M$ satisfies $S \xi=\beta \xi$ for some function $\beta$. If $S Y=\lambda Y$ and if $Y$ is orthogonal to $\xi$, then we have

$$
g\left(\left(\nabla_{\xi} S\right) Y, Y\right) g((\phi A+A \phi) X, W)=0
$$

for any $X, Y$ and $W$ orthogonal to $\xi$.

Proof of Theorem 3.1.

In the following, we suppose that $M$ is not a Hopf hypersurface. We work in an open set where $h_{1} \neq 0$.

Case (I): First we consider the case $g\left(\left(\nabla_{\xi} S\right) Y, Y\right)=0$.

Lemma 3.4. $\beta, \lambda_{1}, \ldots, \lambda_{2 n-2}$ are constant.

Proof. Since the Ricci tensor $S$ is $\eta$-parallel and since $g\left(\left(\nabla_{\xi} S\right) Y, Y\right)=0$, we have

$$
0=g\left(\left(\nabla_{Z} S\right) Y, Y\right)=g\left(\nabla_{Z} S Y, Y\right)-g\left(S \nabla_{Z} Y, Y\right)=Z \lambda
$$

for any tangent vector field Z. So we see that $\lambda_{1}, \ldots, \lambda_{2 n-2}$ are constant. On the other hand, since $\beta=\lambda_{1}-3 c$, we see that $\beta$ is also constant.

Lemma 3.5. If $\lambda_{i} \neq \lambda_{j}, i, j=1, \ldots, 2 n-2$, then we have $g\left(\nabla_{X} e_{i}, e_{j}\right)=0$ for any $X$ orthogonal to $\xi$.

Proof. Since we have $S e_{i}=\lambda_{i} e_{i}$ and $S e_{j}=\lambda_{j} e_{j}$ and since $S$ is $\eta$-parallel, we obtain

$$
0=g\left(\left(\nabla_{X} S\right) e_{i}, e_{j}\right)=\left(\lambda_{i}-\lambda_{j}\right) g\left(\nabla_{X} e_{i}, e_{j}\right) .
$$

If $\lambda_{1}=\cdots=\lambda_{2 n-2}=\lambda$, then $M$ is pseudo-Einstein, i.e., $S X=\lambda X+(\beta-\lambda) \eta(X) \xi$, and so it is a Hopf hypersurface (see [Kon 1979]).

Suppose that $M$ is non-Hopf and that there exist $\lambda_{t}$ and $\lambda_{j}, t, j \geq 2$, satisfying $\lambda_{1} \neq \lambda_{t}$ and $\lambda_{t} \neq \lambda_{j}$. By Lemma 3.5,

$$
\begin{aligned}
g\left(\nabla_{j} \nabla_{t} e_{t}, e_{j}\right) & =-g\left(\nabla_{e_{t}} e_{t}, \nabla_{e_{j}} e_{j}\right) \\
& =-g\left(\nabla_{e_{t}} e_{t}, \xi\right)\left(\xi, \nabla_{e_{j}} e_{j}\right)-\sum_{k} g\left(\nabla_{e_{t}} e_{t}, e_{k}\right) g\left(e_{k}, \nabla_{e_{j}} e_{j}\right) \\
& =-g\left(e_{t}, \phi A e_{t}\right) g\left(\phi A e_{j}, e_{j}\right)=0, \\
g\left(\nabla_{t} \nabla_{j} e_{t}, e_{j}\right) & =-g\left(\nabla_{e_{j}} e_{t}, \nabla_{e_{t}} e_{j}\right)=-g\left(\nabla_{e_{j}} e_{t}, \xi\right) g\left(\xi, \nabla_{e_{t}} e_{g}\right) \\
& =-g\left(e_{t}, \phi A e_{j}\right) g\left(\phi A e_{t}, e_{j}\right)=-a_{j} a_{t} g\left(e_{t}, \phi e_{j}\right) g\left(\phi e_{t}, e_{j}\right) .
\end{aligned}
$$

On the other hand, from (8),

$$
\left(a_{1}-a_{t}\right) g\left(\nabla_{e_{j}} e_{1}, e_{t}\right)+\left(a_{t}-a_{j}\right) g\left(\nabla_{e_{1}} e_{j}, e_{t}\right)+a_{j} h_{1} g\left(\phi e_{j}, e_{t}\right)=0 .
$$

From Lemma 3.5, we have $g\left(\nabla_{e_{j}} e_{1}, e_{t}\right)=0, g\left(\nabla_{e_{1}} e_{j}, e_{t}\right)=0$. Since $h_{1} \neq 0$,

$$
a_{j} g\left(\phi e_{j}, e_{t}\right)=0 \text {, }
$$


from which we obtain

$$
g\left(\nabla_{e_{t}} \nabla_{e_{j}} e_{t}, e_{j}\right)=0
$$

Moreover, we have

$$
\begin{aligned}
g\left(\nabla_{\left[e_{j}, e_{t}\right]} e_{t}, e_{j}\right) & =g\left(\nabla_{\xi} e_{t}, e_{j}\right) g\left(\xi,\left[e_{j}, e_{t}\right]\right) \\
& =g\left(\nabla_{\xi} e_{t}, e_{j}\right)\left(-g\left(\phi A e_{j}, e_{t}\right)+g\left(\phi A e_{t}, e_{j}\right)\right) \\
& =g\left(\nabla_{\xi} e_{t}, e_{j}\right)\left(a_{t}-a_{j}\right) g\left(\phi e_{t}, e_{j}\right) \\
& =g\left(\nabla_{\xi} e_{t}, e_{j}\right) a_{t} g\left(\phi e_{t}, e_{j}\right) .
\end{aligned}
$$

Using (12), we see that

$$
\left(c+a_{j} \alpha-a_{j} a_{t}\right) g\left(\phi e_{j}, e_{t}\right)+h_{1} g\left(\nabla_{e_{j}} e_{1}, e_{t}\right)+\left(a_{t}-a_{j}\right) g\left(\nabla_{\xi} e_{j}, e_{t}\right)=0 .
$$

From these equations, we obtain

$$
\operatorname{cg}\left(\phi e_{j}, e_{t}\right)^{2}+a_{t} g\left(\phi e_{j}, e_{t}\right) g\left(\nabla_{\xi} e_{j}, e_{t}\right)=0 .
$$

Hence we have

$$
g\left(\nabla_{\left[e_{j}, e_{t}\right]} e_{t}, e_{j}\right)=-c g\left(\phi e_{j}, e_{t}\right)^{2} .
$$

Therefore,

$$
g\left(R\left(e_{j}, e_{t}\right) e_{t}, e_{j}\right)=c g\left(\phi e_{j}, e_{t}\right)^{2} .
$$

On the other hand, the equation of Gauss implies

$$
g\left(R\left(e_{j}, e_{t}\right) e_{t}, e_{j}\right)=c+3 c g\left(\phi e_{j}, e_{t}\right)^{2}+a_{t} a_{j} .
$$

From these equations, we have

$$
c\left(1+2 g\left(\phi e_{j}, e_{t}\right)^{2}\right)+a_{t} a_{j}=0 .
$$

Sine $c \neq 0$, we see that $a_{t} \neq 0$ and $a_{j} \neq 0$. Thus $g\left(\phi e_{j}, e_{t}\right)=0$ and $c+a_{t} a_{j}=0$. So we can represent $A$ as

$$
A=\left(\begin{array}{cccccccc}
\alpha & h_{1} & & & & & \\
h_{1} & a_{1} & & & & & \\
& & a & & & & \\
& & & \ddots & & & & \\
& & & & a & & & \\
& & & & b & & \\
& & & & & \ddots & \\
& & & & & & b
\end{array}\right)
$$

by setting $a=a_{j}, b=a_{t}$ and taking a suitable permutation of $\left\{e_{2}, \ldots, e_{2 n-2}\right\}$. 
Suppose there exist $j$ and $t$ such that $g\left(\phi e_{j}, e_{1}\right) \neq 0$ and $g\left(\phi e_{t}, e_{1}\right) \neq 0$. Then $\phi e_{j}$ and $\phi e_{t}$ satisfy

$$
\begin{aligned}
& \phi e_{j}=\sum_{k} g\left(\phi e_{j}, e_{k}\right) e_{k}+g\left(\phi e_{j}, e_{1}\right) e_{1}, \quad A e_{k}=a e_{k}, \\
& \phi e_{t}=\sum_{l} g\left(\phi e_{t}, e_{l}\right) e_{l}+g\left(\phi e_{t}, e_{1}\right) e_{1}, \quad A e_{l}=b e_{l} .
\end{aligned}
$$

So we have

$$
0=g\left(\phi e_{j}, \phi e_{t}\right)=g\left(\phi e_{j}, e_{1}\right) g\left(\phi e_{t}, e_{1}\right),
$$

from which we see that $g\left(\phi e_{j}, e_{1}\right)=0$ or $g\left(\phi e_{t}, e_{1}\right)=0$, and hence $A \phi e_{1}=a \phi e_{1}$ or $A \phi e_{1}=b \phi e_{1}$.

When $A \phi e_{1}=a \phi e_{1}$, we have $A \phi e_{t}=b \phi e_{t}$. By (4),

$$
\left(b-a_{1}\right) g\left(\nabla_{e_{t}} \phi e_{t}, e_{1}\right)-\left(b-a_{1}\right) g\left(\nabla_{\phi e_{t}} e_{t}, e_{1}\right)+2 h_{1} b g\left(\phi e_{t}, \phi e_{t}\right)=0 .
$$

Thus we obtain $b=0$, which contradicts $c+a b=0$ and $c \neq 0$. By a similar computation, the case $A \phi e_{1}=b \phi e_{1}$ does not occur.

Next we consider the case $\lambda_{2}=\cdots=\lambda_{2 n-2} \neq \lambda_{1}$. We set $\lambda=\lambda_{j}, j=2, \ldots, 2 n-2$. From Lemma 3.5, we have $g\left(\nabla_{X} e_{1}, e_{i}\right)=0, i \geq 2$, for any $X$ orthogonal to $\xi$.

By (4) and (5),

$$
h_{1}\left(a_{i}+a_{j}\right) g\left(\phi e_{i}, e_{j}\right)=0, \quad\left(2 c-2 a_{i} a_{j}+\alpha\left(a_{i}+a_{j}\right)\right) g\left(\phi e_{i}, e_{j}\right)=0 .
$$

Since $a_{j}$ satisfies

$$
\lambda=(2 n+1) c+\operatorname{tr} A \cdot a_{j}-a_{j}^{2},
$$

we can represent $A$ as

$$
A=\left(\begin{array}{cccccccc}
\alpha & h_{1} & & & & & \\
h_{1} & a_{1} & & & & & \\
& & a & & & & \\
& & & \ddots & & & \\
& & & & a & & & \\
& & & & b & & \\
& & & & & \ddots & \\
& & & & & & b
\end{array}\right)
$$

by taking a suitable permutation of $\left\{e_{2}, \ldots, e_{2 n-2}\right\}$.

There exist $i$ and $j$ satisfying $g\left(\phi e_{i}, e_{j}\right) \neq 0$. Therefore, using $h_{1} \neq 0$,

$$
a_{i}+a_{j}=0, \quad 2 c-2 a_{i} a_{j}+\alpha\left(a_{i}+a_{j}\right)=0 .
$$

We notice that $\operatorname{tr} A=a_{1}+\alpha$ and $\sum_{j=2}^{2 n-2} a_{j}=k a+l b=0$, where $k$ and $l$ are the multiplicities of $a$ and $b$, respectively. 
When $a_{i}=a_{j}=a$, then we have $a_{i}+a_{j}=2 a=0$. Combining this with the above equations, we obtain $b=0$ and $c=0$. This is a contradiction. Similarly, the case $a_{i}=a_{j}=b$ does not occur.

Next, when $a_{i}=a, a_{j}=b$ and $a=b$, we have $a=b=0$ and $c=0$. This is a contradiction.

Finally we consider the case $a_{i}=a, a_{j}=b$ and $a \neq b$. Then we have $a=-b \neq 0$. Since $k a+l b=0$, we obtain $k=l$. This contradicts the fact that $M$ is an odddimensional real hypersurface.

Case (II): Next we consider the case

$$
g((\phi A+A \phi) X, W)=0
$$

for any $X$ and $W$ orthogonal to $\xi$.

Since $\left\{\xi, \phi e_{1}, \ldots, \phi e_{2 n-2}\right\}$ is an orthonormal basis of the tangent space, we have

$$
\begin{aligned}
\operatorname{tr} A & =g(A \xi, \xi)+\sum_{i=1}^{2 n-2} g\left(A \phi e_{i}, \phi e_{i}\right) \\
& =\alpha-\sum_{i=1}^{2 n-2} g\left(\phi A e_{i}, \phi e_{i}\right)=\alpha-\sum_{i=1}^{2 n-2} g\left(A e_{i}, e_{i}\right) .
\end{aligned}
$$

Since tr $A=\alpha+\sum_{i=1}^{2 n-2} g\left(A e_{i}, e_{i}\right)$, we obtain $\sum_{i=1}^{2 n-2} g\left(A e_{i}, e_{i}\right)=0$ and $\operatorname{tr} A=\alpha$. On the other hand, from $\operatorname{tr} A=a_{1}+\alpha$, we have $a_{1}=0$. Substituting $X=e_{1}$ in (18), we see that $g\left(A \phi e_{1}, W\right)=0$ for any $W$ orthogonal to $\xi$. Since

$$
g\left(A \phi e_{1}, \xi\right)=g\left(\phi e_{1}, A \xi\right)=0,
$$

we have $A \phi e_{1}=0$. Without loss of generality, we can set $\phi e_{1}=e_{2}$. From (13) and (17), we obtain

$$
\begin{gathered}
\left(e_{2} h_{1}\right)=c+h_{1}^{2}, \\
\left(c-h_{1}^{2}\right)+h_{1} g\left(\nabla_{e_{1}} e_{2}, e_{1}\right)=0 .
\end{gathered}
$$

On the other hand, since $S$ is $\eta$-parallel, putting $X=Y=e_{1}$ and $Z=e_{2}$ into (2), we have

$$
0=\operatorname{tr} A g\left(\left(\nabla_{e_{1}} A\right) e_{1}, e_{2}\right)-g\left(\left(\nabla_{e_{1}} A\right) A e_{1}, e_{2}\right)=h_{1}^{2} g\left(e_{1}, \nabla_{e_{1}} e_{2}\right) .
$$

Since $h_{1} \neq 0$, we have $g\left(\nabla_{e_{1}} e_{2}, e_{1}\right)=0$. Combining this with (20), we see that $h_{1}^{2}=c$. This contradicts (19), finishing the proof.

We remark that Suh [1990] and Maeda [2013] classified Hopf hypersurfaces of nonflat complex space forms with $\eta$-parallel Ricci tensor. 


\section{Ruled real hypersurfaces}

In the previous sections, under the condition that the Ricci tensor $S$ of $M$ satisfies $S \xi=\beta \xi$, we gave sufficient conditions for $M$ to be a Hopf hypersurface with respect to the covariant derivative of the Ricci tensor of $S$. The purpose of this section is to give a condition on the Ricci tensor for $M$ to be a ruled real hypersurface.

Theorem 4.1. Let $M$ be a real hypersurface of $M^{n}(c), c \neq 0$. If the Ricci tensor $S$ of $M$ satisfies $S \xi=\beta \xi$ for some function $\beta$ and if $g\left(\left(\nabla_{X} S\right) Y, \xi\right)=0$ for any vector fields $X$ and $Y$ orthogonal to $\xi$, then $M$ is a ruled real hypersurface.

Proof. To prove Theorem 4.1, we need the following proposition:

Proposition 4.2. Let $M$ be a real hypersurface of $M^{n}(c), c \neq 0$. If the Ricci tensor $S$ of $M$ satisfies $S \xi=\beta \xi$ for some function $\beta$ and if $g\left(\left(\nabla_{X} S\right) Y, \xi\right)=0$ for any vector fields $X$ and $Y$ orthogonal to $\xi$, then $M$ is not Hopf.

Proof. Suppose that $M$ is a Hopf hypersurface. Then we have $A \xi=\alpha \xi$, and hence $S \xi=\beta \xi$. We note that $\alpha$ is constant. Therefore, we have

$$
\begin{aligned}
g\left(\left(\nabla_{X} S\right) Y, \xi\right) & =g\left(\left(\nabla_{X} S\right) \xi, Y\right) \\
& =g\left(\nabla_{X} S \xi, Y\right)-g(S \phi A X, Y) \\
& =\beta g(\phi A X, Y)-g(\phi A X, S Y)
\end{aligned}
$$

for any $X$ and $Y$ orthogonal to $\xi$. We take an orthonormal basis $\left\{\xi, e_{1}, \ldots, e_{2 n-2}\right\}$ that satisfies $e_{2 i}=\phi e_{2 i-1}, i=1, \ldots, n-1$, and set $A e_{t}=a_{t} e_{t}, t=1, \ldots, 2 n-2$. Then we have $A \phi e_{t}=\bar{a}_{t} \phi e_{t}$ since $M$ is Hopf. Then the Ricci operator $S$ satisfies $S \xi=\beta \xi$ and $S e_{t}=\lambda_{t} e_{t}, t=1, \ldots, 2 n-2$, where

$$
\beta=(2 n-2) c+\operatorname{tr} A \cdot \alpha-\alpha^{2}, \quad \lambda_{t}=(2 n+1) c+\operatorname{tr} A \cdot a_{t}-a_{t}^{2} .
$$

Thus we obtain

$$
0=\left(\beta-\lambda_{t}\right) g\left(\phi A X, e_{t}\right)=-\left(\beta-\lambda_{t}\right) g\left(X, A \phi e_{t}\right)
$$

for any $X$ orthogonal to $\xi$. Since $A \xi=\alpha \xi$, we have $g\left(A \phi e_{t}, \xi\right)=0$. From these equations, we have:

Lemma 4.3. If $\beta \neq \lambda_{t}$, then $A \phi e_{t}=0$, that is, $\bar{a}_{t}=0$.

We suppose $\beta \neq \lambda_{t}$. Then, from (1), we have

$$
\overline{\lambda_{t}}=g\left(S \phi e_{t}, \phi e_{t}\right)=(2 n+1) c .
$$

Using Proposition A and $c \neq 0$, we have $\alpha \neq 0$ and

$$
a_{t}=-\frac{2 c}{\alpha} \text {. }
$$


If $\beta \neq \lambda_{t}$ and $\beta \neq \bar{\lambda}_{t}=g\left(S \phi e_{t}, \phi e_{t}\right)$, then we have $a_{t}=\bar{a}_{t}=0$. This is a contradiction. Thus we obtain:

Lemma 4.4. If $\beta \neq \lambda_{t}$, then $\beta=\bar{\lambda}_{t}=(2 n+1) c$.

Since $M$ is not Einstein, there exists a $t$ such that $\beta \neq \lambda_{t}$. So we see that $\lambda_{t}$ satisfies $\beta=\lambda_{t}=\bar{\lambda}_{t}$ or $\beta=\bar{\lambda}_{t} \neq \lambda_{t}$.

When $\beta=\lambda_{t}=\bar{\lambda}_{t}$, since $\beta=(2 n+1) c$, we have

$$
0=a_{t}\left(\operatorname{tr} A-a_{t}\right) .
$$

So we obtain $a_{t}=0$ or $a_{t}=\operatorname{tr} A$. If $a_{t}=0$, then $\bar{a}_{t}=-2 c / \alpha$. There exists an $s$ that satisfies $\lambda_{s} \neq \beta$, and hence $a_{s}=-2 c / \alpha$. Thus we have

$$
\beta \neq \lambda_{s}=(2 n+1) c+\operatorname{tr} A\left(\frac{-2 c}{\alpha}\right)-\left(-\frac{2 c}{\alpha}\right)^{2} .
$$

Thus $\bar{\lambda}_{t}=\lambda_{s} \neq \beta$. This is a contradiction. So we see that $a_{t}=\operatorname{tr} A \neq 0$. In the following, we set $a=a_{t}=\operatorname{tr} A$. Since $a_{t}=\bar{a}_{t}=\operatorname{tr} A$, we have

$$
(2 a-\alpha) a=(\alpha a+2 c) \text {. }
$$

Thus $a$ satisfies $a^{2}-\alpha a-c=0$, and hence $a$ turns to be constant. In the following, we set $a_{1}=-2 c / \alpha$ and $\bar{a}_{1}=a_{2}=0$.

Next we compute $g\left(R\left(e_{1}, e_{2}\right) e_{2}, e_{1}\right)$. By the equation of Gauss,

$$
g\left(R\left(e_{1}, e_{2}\right) e_{2}, e_{1}\right)=g\left(R\left(e_{1}, \phi e_{1}\right) \phi e_{1}, e_{1}\right)=4 c .
$$

Using (7), $a_{1} g\left(\nabla_{e_{2}} e_{1}, e_{2}\right)=0$. Since $a_{1} \neq 0$, we have $g\left(\nabla_{e_{2}} e_{2}, e_{1}\right)=0$. Moreover,

$$
g\left(\nabla_{e_{2}} e_{2}, e_{2}\right)=0, \quad g\left(\nabla_{e_{2}} e_{2}, \xi\right)=-g\left(e_{2}, \phi A e_{2}\right)=0 .
$$

When $k \geq 3$, by (6),

$$
a_{k} g\left(\nabla_{e_{2}} e_{2}, e_{k}\right)=0 \text {. }
$$

When $a_{k} \neq 0$, we have $g\left(\nabla_{e_{2}} e_{2}, e_{k}\right)=0$. By (10), $g\left(\nabla_{e_{1}} e_{1}, e_{2}\right)=0$. Moreover,

$$
g\left(\nabla_{e_{1}} e_{1}, e_{1}\right)=0, \quad g\left(\nabla_{e_{1}} e_{1}, \xi\right)=0 .
$$

Since $k \geq 3$, by (10) and the fact that $a_{1}$ is constant,

$$
\left(a_{1}-a_{k}\right) g\left(\nabla_{e_{1}} e_{k}, e_{1}\right)=0 .
$$

By $a_{1} \neq 0$, if $a_{k}=0$, then $g\left(\nabla_{e_{1}} e_{1}, e_{k}\right)=0$. Thus we have

$$
\sum_{k=1}^{2 n-2} g\left(\nabla_{e_{1}} e_{1}, e_{k}\right) g\left(e_{k}, \nabla_{e_{2}} e_{2}\right)=0 \text {. }
$$


So we have

$$
\begin{aligned}
g\left(\nabla_{e_{1}} \nabla_{e_{2}} e_{2}, e_{1}\right) & =e_{1} g\left(\nabla_{e_{2}} e_{2}, e_{1}\right)-g\left(\nabla_{e_{2}} e_{2}, \nabla_{e_{1}} e_{1}\right) \\
& =-\sum_{k} g\left(\nabla_{e_{2}} e_{2}, e_{k}\right) g\left(e_{k}, \nabla_{e_{1}} e_{1}\right)=0, \\
g\left(\nabla_{e_{2}} \nabla_{e_{1}} e_{2}, e_{1}\right) & =e_{2} g\left(\nabla_{e_{1}} e_{2}, e_{1}\right)-g\left(\nabla_{e_{1}} e_{2}, \nabla_{e_{2}} e_{1}\right)=-g\left(\nabla_{e_{1}} \phi e_{1}, \nabla_{e_{2}} e_{1}\right) \\
& =g\left(\nabla_{e_{1}} e_{1}, \phi \nabla_{e_{2}} e_{1}\right)=g\left(\nabla_{e_{1}} e_{1}, \nabla_{e_{2}} e_{2}\right)=0,
\end{aligned}
$$

and

$$
\begin{aligned}
g & \left(\nabla_{\left[e_{1}, e_{2}\right]} e_{2}, e_{1}\right) \\
& =g\left(\nabla_{\xi} e_{2}, e_{1}\right) g\left(\xi,\left[e_{1}, e_{2}\right]\right)+\sum_{k \geq 3} g\left(\nabla_{k} e_{2}, e_{1}\right) g\left(e_{k},\left[e_{1}, e_{2}\right]\right) \\
& =-a_{1} g\left(\nabla_{\xi} e_{2}, e_{1}\right)+\sum_{k \geq 3} g\left(\nabla_{e_{k}} e_{2}, e_{1}\right) g\left(e_{k}, \nabla_{e_{1}} e_{2}\right)-\sum_{k \geq 3} g\left(\nabla_{e_{k}} e_{2}, e_{1}\right) g\left(e_{k}, \nabla_{e_{2}} e_{1}\right) .
\end{aligned}
$$

By (13),

$$
a_{1} g\left(\nabla_{\xi} e_{2}, e_{1}\right)=c .
$$

Using (4), we have

$$
g\left(\nabla_{e_{k}} e_{2}, e_{1}\right)=\frac{a_{k}-a_{1}}{a_{1}} g\left(\nabla_{e_{2}} e_{1}, e_{k}\right) .
$$

On the other hand, by (8),

$$
g\left(\nabla_{e_{k}} e_{2}, e_{1}\right)=\frac{a_{k}}{a_{1}} g\left(\nabla_{e_{1}} e_{2}, e_{k}\right) .
$$

So we obtain

$$
\begin{aligned}
\sum_{k \geq 3} g\left(\nabla_{e_{k}} e_{2}, e_{1}\right)\left(e_{k}, \nabla_{e_{1}} e_{2}\right)-\sum \sum_{k \geq 3} g\left(\nabla_{e_{k}} e_{2}, e_{1}\right) g\left(e_{k}, \nabla_{e_{2}} e_{1}\right) \\
=\sum \frac{\left(a_{k}-a_{1}\right)}{a_{1}} g\left(\nabla_{e_{2}} e_{1}, e_{k}\right) g\left(e_{k}, \nabla_{e_{1}} e_{2}\right)-\sum \frac{a_{k}}{a_{1}} g\left(\nabla_{e_{1}} e_{2}, e_{k}\right)\left(e_{k}, \nabla_{e_{2}} e_{1}\right) \\
=-\sum g\left(\nabla_{e_{2}} e_{1}, e_{k}\right) g\left(e_{k}, \nabla_{e_{1}} e_{2}\right) \\
=-\sum g\left(\nabla_{e_{2}} e_{1}, \phi e_{k}\right) g\left(\phi e_{k}, \nabla_{e_{1}} e_{2}\right) \\
=\sum g\left(\nabla_{e_{2}} e_{2}, e_{k}\right) g\left(e_{k}, \nabla_{e_{1}} e_{1}\right)=0 .
\end{aligned}
$$

Thus we have

$$
g\left(R\left(e_{1}, e_{2}\right) e_{2}, e_{1}\right)=c,
$$

from which we obtain $c=0$. This is a contradiction. Hence we see that $M$ is not Hopf. Thus we have proven Proposition 4.2. 
From Proposition 4.2, if $g\left(\left(\nabla_{X} S\right) Y, \xi\right)=0$ for $X, Y \in H$, then $M$ is not Hopf. In the following, we suppose that $M$ is not Hopf, that is, $h_{1} \neq 0$. Then, by Lemma 2.2, we can take an orthonormal basis $\left\{\xi, e_{1}, \ldots, e_{2 n-2}\right\}$ such that

$$
\begin{gathered}
A \xi=\alpha \xi+h_{1} e_{1}, \quad A e_{1}=a_{1} e_{1}+h_{1} \xi, \quad A e_{j}=a_{j} e_{j}, \quad j=2, \ldots, 2 n-2, \\
\operatorname{tr} A=\alpha+a_{1}, \quad a_{2}+\cdots+a_{2 n-2}=0 .
\end{gathered}
$$

Then we have

$$
\begin{aligned}
\beta & =g(S \xi, \xi)=(2 n-2) c+\left(a_{1} \alpha-h_{1}^{2}\right), \\
\lambda_{1} & =g\left(S e_{1}, e_{1}\right)=(2 n+1) c+\left(a_{1} \alpha-h_{1}^{2}\right), \\
\lambda_{j} & =g\left(S e_{j}, e_{j}\right)=(2 n+1) c+\operatorname{tr} A \cdot a_{j}-a_{j}^{2}, \quad j \geq 2 .
\end{aligned}
$$

By the assumption, for any $X$ and $Y$ orthogonal to $\xi$,

$$
0=g\left(\left(\nabla_{X} S\right) \xi, Y\right)=g\left(\nabla_{X} S \xi, Y\right)-g(S \phi A X, Y) .
$$

We set $S Y=\lambda Y$. Then we have

$$
0=(\beta-\lambda) g(\phi A X, Y) .
$$

Since $\beta \neq \lambda_{1}$, we see that

$$
g\left(\phi A X, e_{1}\right)=-g\left(A X, \phi e_{1}\right)=-g\left(X, A \phi e_{1}\right)=0
$$

for any $X \in H$. We also have $g\left(\xi, A \phi e_{1}\right)=0$. Thus we have $A \phi e_{1}=0$. In the following, we set $\phi e_{1}=e_{2}$. Then we have

$$
0=\left(\beta-\lambda_{2}\right) g\left(\phi A e_{1}, e_{2}\right)=\left(-3 c+a_{1} \alpha-h_{1}^{2}\right) a_{1} .
$$

Lemma 4.5. If $h_{1} \neq 0$, then $a_{2}=0$. Moreover, $a_{1}=0$ or $a_{1} \alpha-h_{1}^{2}=3 c$.

Case (I): Suppose $a_{1}=0$.

Since $a_{1}=a_{2}=0$, (13) implies

$$
\left(e_{2} h_{1}\right)=c+h_{1}^{2} .
$$

If $\beta=(2 n+1) c=\lambda_{2}$, then $h_{1}^{2}=-3 c$ and $e_{2} h_{1}=0$. Then we have $h_{1}^{2}=-c$ and $c=0$. This is a contradiction. So we have:

Lemma 4.6. If $a_{1}=0$, then $\beta \neq(2 n+1) c=\lambda_{2}$.

For any $X \in H$, we see that

$$
\left(\beta-\lambda_{k}\right) g\left(\phi A X, e_{k}\right)=0, \quad k \geq 3 .
$$

If $\beta \neq \lambda_{k}$, then $g\left(A \phi e_{k}, X\right)=0$, and moreover $g\left(A \phi e_{k}, \xi\right)=0$. This shows that $A \phi e_{k}=0$ and that $\phi e_{k}$ is a principal vector of $A$. We set

$$
\bar{\lambda}_{k}=g\left(S \phi e_{k}, \phi e_{k}\right) .
$$


Since $a_{1} \alpha-h_{1}^{2} \neq 3 c$, we have $\bar{\lambda}_{k}=(2 n+1) c \neq \beta$. Then, from

$$
\left(\beta-\bar{\lambda}_{k}\right) g\left(\phi A X, \phi e_{k}\right)=0,
$$

we have $g\left(A e_{k}, X\right)=0$. We also have $g\left(A e_{k}, \xi\right)=0$ since $k \geq 3$. Hence we obtain $A e_{k}=0$ for $e_{k}$ satisfying $\beta \neq \lambda_{k}$.

We next consider the case $\beta=\lambda_{j}$ for some $j \geq 3$. If $\beta=\lambda_{j}=\lambda_{i}$, then

$$
\beta=(2 n+1) c+\operatorname{tr} A \cdot a_{j}-a_{j}^{2}=(2 n+1) c+\operatorname{tr} A \cdot a_{i}-a_{i}^{2} .
$$

Therefore, at most two $a_{j}$ are different. By this equation, we have

$$
0=\left(a_{j}-a_{i}\right)\left(\operatorname{tr} A-\left(a_{j}+a_{i}\right)\right) .
$$

If $a_{j}=a_{i}=a$ for all $j$ and $i$, then (21) implies $\sum a_{j}=0$. Thus we have all $a_{j}=0$, $j=2, \ldots, 2 n-2$. Since $a_{1}=0, M$ is a ruled real hypersurface.

Let us suppose that two $a_{j}$ are different. We set

$$
T_{a}=\left\{X \mid A X=a X, X \in H_{x}\right\}, \quad T_{b}=\left\{X \mid A X=b X, X \in H_{x}\right\},
$$

where $\beta=\lambda_{a}=\lambda_{b}, a \neq b$. We notice $\operatorname{tr} A=a+b$. If $a=0$ or $b=0$, then, by (21), $a=b=0$. This contradicts the assumption that $a \neq b$. So we obtain $a \neq 0$ and $b \neq 0$. We notice that $\operatorname{dim} T_{a}+\operatorname{dim} T_{b}$ is even number.

Let $e_{i}, e_{j} \in T_{a}$. By (8) and (12),

$$
\begin{array}{r}
-a g\left(\nabla_{e_{i}} e_{1}, e_{j}\right)+a h_{1} g\left(\phi e_{i}, e_{j}\right)=0, \\
\left(c+a \alpha-a^{2}\right) g\left(\phi e_{i}, e_{j}\right)+h_{1} g\left(\nabla_{e_{i}} e_{1}, e_{j}\right)=0 .
\end{array}
$$

From these, we obtain

$$
\left(c+a \alpha-a^{2}+h_{1}^{2}\right) g\left(\phi e_{i}, e_{j}\right)=0 .
$$

If there exist $e_{i}$ and $e_{j}$ such that $g\left(\phi e_{i}, e_{j}\right) \neq 0$, then

$$
c+a \alpha-a^{2}+h_{1}^{2}=0 .
$$

On the other hand, we have

$$
\beta=(2 n-2) c-h_{1}^{2}=(2 n+1) c+\operatorname{tr} A \cdot a-a^{2} .
$$

Since $\operatorname{tr} A=\alpha+a_{1}=\alpha$, we have

$$
3 c+\alpha a-a^{2}+h_{1}^{2}=0 .
$$

Therefore, we have $2 c=0$. This contradicts $c \neq 0$. Hence $g\left(\phi e_{i}, e_{j}\right)=0$ for all $e_{i}$ and $e_{j}$ of $T_{a}$. So we have $\phi T_{a} \subset T_{b}$. Similarly, we also have $\phi T_{b} \subset T_{a}$. Consequently, we see that

$$
\phi T_{a}=T_{b}, \quad \phi T_{b}=T_{a} .
$$


If $\operatorname{dim} T_{a}=\operatorname{dim} T_{b}=1$, then $\phi T_{a}=T_{b}$. We see that if $A e_{j}=a e_{j}$, then $A \phi e_{j}=b \phi e_{j}$ and $a+b=\operatorname{tr} A$. From (21), we have $a+b=0$ and $\operatorname{tr} A=0$. Therefore, we obtain $\operatorname{tr} A=\alpha=0$.

We will prove that there is no real hypersurface that satisfies

$$
a+b=0, \quad \alpha=0, \quad a_{1}=0, \quad a_{2}=0, \quad \operatorname{tr} A=0,
$$

and also

$$
a^{2}-h_{1}^{2}=3 c
$$

By (5),

$$
\left(2 c+2 a^{2}\right) g\left(\phi e_{i}, \phi e_{i}\right)-h_{1} g\left(\nabla_{e_{i}} \phi e_{i}, e_{1}\right)+h_{1} g\left(\nabla_{\phi e_{i}} e_{i}, e_{1}\right)=0 .
$$

On the other hand, we have

$$
g\left(\nabla_{e_{i}} \phi e_{i}, e_{1}\right)=g\left(\phi \nabla_{e_{i}} e_{i}, e_{1}\right)=-g\left(\nabla_{e_{i}} e_{i}, e_{2}\right) .
$$

By (6),

$$
\left(a_{2}-a_{i}\right) g\left(\nabla_{e_{i}} e_{2}, e_{i}\right)-\left(e_{2} a_{i}\right)=0 .
$$

Using $a_{2}=0$ and $a_{i}=a$, we obtain

$$
\operatorname{ag}\left(\nabla_{e_{i}} e_{i}, e_{2}\right)=\left(e_{2} a\right) .
$$

From this equation and $a \neq 0$, we have

$$
g\left(\nabla_{e_{i}} e_{i}, e_{2}\right)=\frac{\left(e_{2} a\right)}{a} .
$$

On the other hand,

$$
g\left(\nabla_{\phi e_{i}} e_{i}, e_{1}\right)=g\left(\phi \nabla_{\phi e_{i}} e_{i}, \phi e_{1}\right)=g\left(\nabla_{\phi e_{i}} \phi e_{i}, e_{2}\right) .
$$

By (6), we obtain

$$
\left(a_{2}+a\right) g\left(\nabla_{\phi e_{i}} e_{2}, \phi e_{i}\right)+\left(e_{2} a\right)=0,
$$

and hence

$$
g\left(\nabla_{\phi e_{i}} \phi e_{i}, e_{2}\right)=\frac{\left(e_{2} a\right)}{a} .
$$

Substituting these equations into (22), we get

$$
2\left(c+a^{2}\right)+h_{1} \frac{\left(e_{2} a\right)}{a}+h_{1} \frac{\left(e_{2} a\right)}{a}=0 .
$$

Thus we have

$$
\left(c+a^{2}\right) a=-h_{1}\left(e_{2} a\right) .
$$

On the other hand, since $a^{2}-h_{1}^{2}=3 c$,

$$
a\left(e_{2} a\right)=h_{1}\left(e_{2} h_{1}\right) \text {. }
$$


Since $a_{1}=a_{2}=0$, by (13), we have

$$
e_{2} h_{1}=c+h_{1}^{2},
$$

from which we obtain

$$
e_{2} a=\frac{h_{1}}{a}\left(c+h_{1}^{2}\right) .
$$

Substituting this into (23), we get

$$
\left(c+a^{2}\right) a=-\frac{h_{1}^{2}}{a}\left(c+h_{1}^{2}\right)=-\frac{1}{a}\left(a^{2}-3 c\right)\left(a^{2}-2 c\right) .
$$

Thus we obtain

$$
\left(a^{2}-c\right)^{2}+2 c^{2}=0
$$

So we have $c=0$. This is a contradiction. Consequently, if $a_{1}=0$, then $M$ is a ruled real hypersurface.

Case (II): Suppose $a_{1} \neq 0$.

We notice that $a_{2}=0$ and $\alpha a_{1} h_{1}^{2}=3 c$ by Lemma 4.5. So we have

$$
\left(X a_{1}\right) \alpha+a_{1}(X \alpha)-2 h_{1}\left(X h_{1}\right)=0
$$

for any tangent vector field $X$.

Lemma 4.7. $\nabla_{e_{1}} e_{1}$ and $\nabla_{e_{2}} e_{2}$ are perpendicular to $\xi, e_{1}$ and $e_{2}$.

Proof. By (14),

$$
\left(e_{2} \alpha\right)=\alpha h_{1}+h_{1} g\left(\nabla_{\xi} e_{1}, e_{2}\right)
$$

By (10),

$$
\left(e_{2} a_{1}\right)=a_{1} h_{1}+a_{1} g\left(\nabla_{e_{1}} e_{1}, e_{2}\right) .
$$

Substituting these into (24), we get

$$
2 a_{1} \alpha h_{1}+\alpha a_{1} g\left(\nabla_{e_{1}} e_{1}, e_{2}\right)+a_{1} h_{1} g\left(\nabla_{\xi} e_{1}, e_{2}\right)-2 h_{1}\left(e_{2} h_{1}\right)=0 .
$$

By (9) and (13),

$$
\begin{aligned}
& \left(e_{2} h_{1}\right)=\left(2 c+\alpha a_{1}\right)+h_{1} g\left(\nabla_{e_{1}} e_{1}, e_{2}\right)=\left(5 c+h_{1}^{2}\right)+h_{1} g\left(\nabla_{e_{1}} e_{1}, e_{2}\right), \\
& \left(e_{2} h_{1}\right)=\left(c+h_{1}^{2}\right)+a_{1} g\left(\nabla_{\xi} e_{1}, e_{2}\right) .
\end{aligned}
$$

From these equations and (24), we have

$$
2 h_{1}\left(a_{1} \alpha-h_{1}^{2}-3 c\right)+\left(a_{1} \alpha-h_{1}^{2}\right) g\left(\nabla_{e_{1}} e_{1}, e_{2}\right)=0 .
$$


Since $a_{1} \alpha-h_{1}^{2}=3 c$, we have

$$
g\left(\nabla_{e_{1}} e_{1}, e_{2}\right)=0
$$

By (7), $a_{1} \neq 0$ and $a_{2}=0$,

$$
g\left(\nabla_{e_{2}} e_{2}, e_{1}\right)=0
$$

Moreover, we have

$$
g\left(\nabla_{e_{2}} e_{2}, \xi\right)=-g\left(e_{2}, \phi A e_{2}\right)=0, \quad g\left(\nabla_{e_{1}} e_{1}, \xi\right)=-g\left(e_{1}, \phi A e_{1}\right)=0
$$

These equations prove our lemma.

Lemma 4.8. Suppose $j \geq 3$. If $a_{j}=0$, then $g\left(\nabla_{e_{1}} e_{1}, e_{j}\right)=0$. If $a_{j} \neq 0$, then $g\left(\nabla_{e_{2}} e_{2}, e_{j}\right)=0$.

Proof. By (6), we have

$$
a_{j} g\left(\nabla_{e_{2}} e_{2}, e_{j}\right)=0, \quad j \geq 3 .
$$

If $a_{j} \neq 0$, then $g\left(\nabla_{e_{2}} e_{2}, e_{j}\right)=0$ for $j \geq 3$. Suppose $a_{j}=0, j \geq 3$. Then, by (10), (14), (9) and (13),

$$
\begin{aligned}
& \left(e_{j} a_{1}\right)=a_{1} g\left(\nabla_{e_{1}} e_{1}, e_{j}\right), \quad\left(e_{j} \alpha\right)=h_{1} g\left(\nabla_{\xi} e_{1}, e_{j}\right), \\
& \left(e_{j} h_{1}\right)=h_{1} g\left(\nabla_{e_{1}} e_{1}, e_{j}\right), \quad\left(e_{j} h_{1}\right)=a_{1} g\left(\nabla_{\xi} e_{1}, e_{j}\right) .
\end{aligned}
$$

Substituting these into (24), we get

$$
\begin{aligned}
0 & =\left(e_{j} a_{1}\right) \alpha+a_{1}\left(e_{j} \alpha\right)-2 h_{1}\left(e_{j} h_{1}\right) \\
& =\alpha a_{1} g\left(\nabla_{e_{1}} e_{1}, e_{j}\right)+a_{1} h_{1} g\left(\nabla_{\xi} e_{1}, e_{j}\right)-h_{1}^{2} g\left(\nabla_{e_{1}} e_{1}, e_{j}\right)-h_{1} a_{1} g\left(\nabla_{\xi} e_{1}, e_{j}\right) \\
& =\left(\alpha a_{1}-h_{1}^{2}\right) g\left(\nabla_{e_{1}} e_{1}, e_{j}\right) .
\end{aligned}
$$

Since $a_{1} \alpha-h_{1}^{2}=3 c$, we have our lemma.

Using these lemmas, we compute $g\left(R\left(e_{1}, e_{2}\right) e_{2}, e_{1}\right)$. We note that $e_{2}=\phi e_{1}$ and $a_{2}=0$. First, we have

$$
\begin{aligned}
g\left(\nabla_{e_{1}} \nabla_{e_{2}} e_{2}, e_{1}\right)= & e_{1} g\left(\nabla_{e_{2}} e_{2}, e_{1}\right)-g\left(\nabla_{e_{2}} e_{2}, \nabla_{e_{1}} e_{1}\right) \\
=- & g\left(\nabla_{e_{2}} e_{2}, \xi\right) g\left(\xi, \nabla_{e_{1}} e_{1}\right)-g\left(\nabla_{e_{2}} e_{2}, e_{1}\right) g\left(e_{1}, \nabla_{e_{1}} e_{1}\right) \\
& \quad-g\left(\nabla_{e_{2}} e_{2}, e_{2}\right) g\left(e_{2}, \nabla_{e_{1}} e_{1}\right)-\sum_{k \geq 3} g\left(\nabla_{e_{2}} e_{2}, e_{j}\right) g\left(e_{j}, \nabla_{e_{1}} e_{1}\right)=0 .
\end{aligned}
$$


Next, we have

$$
\begin{aligned}
g\left(\nabla_{e_{2}} \nabla_{e_{1}} e_{2}, e_{1}\right) & =e_{2} g\left(\nabla_{e_{1}} e_{2}, e_{1}\right)-g\left(\nabla_{e_{1}} e_{2}, \nabla_{e_{2}} e_{1}\right) \\
=- & g\left(\nabla_{e_{1}} e_{2}, \xi\right) g\left(\xi, \nabla_{e_{2}} e_{1}\right)-g\left(\nabla_{e_{1}} e_{2}, e_{1}\right) g\left(e_{1}, \nabla_{e_{2}} e_{1}\right) \\
& \quad-g\left(\nabla_{e_{1}} e_{2}, \xi\right) g\left(\xi, \nabla_{e_{2}} e_{1}\right)-\sum_{k \geq 3} g\left(\nabla_{e_{1}} e_{2}, e_{k}\right) g\left(e_{k}, \nabla_{e_{2}} e_{1}\right) \\
=- & \sum_{k \geq 3} g\left(\nabla_{e_{1}} e_{2}, e_{k}\right) g\left(e_{k}, \nabla_{e_{2}} e_{1}\right)=-\sum_{k \geq 3} g\left(\nabla_{e_{1}} \phi e_{1}, e_{k}\right) g\left(\phi e_{k}, \phi \nabla_{e_{2}} e_{1}\right) \\
= & \sum_{k \geq 3} g\left(\nabla_{e_{1}} e_{1}, \phi e_{k}\right) g\left(\phi e_{k}, \nabla_{e_{2}} e_{2}\right)=\sum_{l \geq 3} g\left(\nabla_{e_{1}} e_{1}, e_{l}\right) g\left(e_{l}, \nabla_{e_{2}} e_{2}\right)=0 .
\end{aligned}
$$

Moreover, we obtain

$$
\begin{aligned}
g\left(\nabla_{\left[e_{1}, e_{2}\right]} e_{2}, e_{1}\right)= & g\left(\nabla_{\xi} e_{2}, e_{1}\right) g\left(\xi,\left[e_{1}, e_{2}\right]\right)+g\left(\nabla_{e_{1}} e_{2}, e_{1}\right) g\left(e_{1},\left[e_{1}, e_{2}\right]\right) \\
& \quad+g\left(\nabla_{e_{2}} e_{2}, e_{1}\right) g\left(e_{2} \cdot\left[e_{1}, e_{2}\right]\right)+\sum_{k \geq 3} g\left(\nabla_{e_{k}} e_{2}, e_{1}\right) g\left(e_{k},\left[e_{1}, e_{2}\right]\right) \\
= & g\left(\nabla_{\xi} e_{2}, e_{1}\right) g\left(\xi, \nabla_{e_{1}} e_{2}\right) \\
& \quad+\sum_{k \geq 3}\left(g\left(\nabla_{e_{k}} e_{2}, e_{1}\right) g\left(e_{k}, \nabla_{e_{1}} e_{2}\right)-g\left(\nabla_{e_{k}} e_{2}, e_{1}\right) g\left(e_{k}, \nabla_{e_{2}} e_{1}\right)\right) .
\end{aligned}
$$

On the other hand, by (8), when $j \geq 3$,

$$
\begin{array}{r}
a_{1} g\left(\nabla_{e_{j}} e_{2}, e_{1}\right)-a_{j} g\left(\nabla_{e_{1}} e_{2}, e_{j}\right)=0, \\
\left(a_{1}-a_{j}\right) g\left(\nabla_{e_{2}} e_{1}, e_{j}\right)+a_{j} g\left(\nabla_{e_{1}} e_{2}, e_{j}\right)=0 .
\end{array}
$$

Thus, if $a_{1}=a_{j}$, then we see that $a_{j} \neq 0$ and hence $g\left(\nabla_{e_{1}} e_{2}, e_{j}\right)=0$ since $a_{1} \neq 0$. Next, when $a_{1} \neq a_{j}$ we have

$$
g\left(\nabla_{e_{2}} e_{1}, e_{j}\right)=-\frac{a_{j}}{\left(a_{1}-a_{j}\right)} g\left(\nabla_{e_{1}} e_{2}, e_{j}\right) .
$$

On the other hand,

$$
g\left(\nabla_{e_{j}} e_{2}, e_{1}\right)=\frac{a_{j}}{a_{1}} g\left(\nabla_{e_{1}} e_{2}, e_{j}\right)=-\frac{\left(a_{1}-a_{j}\right)}{a_{1}} g\left(\nabla_{e_{2}} e_{1}, e_{j}\right) .
$$

So we have

$$
\begin{aligned}
& \sum_{k \geq 3}\left(g\left(\nabla_{e_{k}} e_{2}, e_{1}\right) g\left(e_{k}, \nabla_{e_{1}} e_{2}\right)-g\left(\nabla_{e_{k}} e_{2}, e_{1}\right) g\left(e_{k}, \nabla_{e_{2}} e_{1}\right)\right. \\
& =-\sum_{k \geq 3} g\left(\nabla_{e_{2}} e_{1}, e_{k}\right) g\left(e_{k}, \nabla_{e_{1}} e_{2}\right)=-\sum_{k \geq 3} g\left(\phi \nabla_{e_{2}} e_{1}, e_{k}\right) g\left(\phi e_{k}, \nabla_{e_{1}} e_{2}\right) \\
& =\sum_{l \geq 3} g\left(\nabla_{e_{1}} e_{1}, e_{l}\right) g\left(e_{l}, \nabla_{e_{2}} e_{2}\right)=0 .
\end{aligned}
$$


Thus we obtain

$$
\begin{aligned}
g\left(\nabla_{\left[e_{1}, e_{2}\right]} e_{2}, e_{1}\right) & =g\left(\nabla_{\xi} e_{2}, e_{1}\right) g\left(\xi, \nabla_{e_{1}} e_{2}\right) \\
& =-g\left(\nabla_{\xi} e_{2}, e_{1}\right) g\left(\phi A e_{1}, e_{2}\right)=-a_{1} g\left(\nabla_{\xi} e_{2}, e_{1}\right),
\end{aligned}
$$

and so

$$
g\left(R\left(e_{1}, e_{2}\right) e_{2}, e_{1}\right)=a_{1} g\left(\nabla_{\xi} e_{2}, e_{1}\right) .
$$

On the other hand, by (9),

$$
-\left(2 c+\alpha a_{1}\right)+h_{1} g\left(\nabla_{e_{1}} e_{2}, e_{1}\right)+\left(e_{2} h_{1}\right)=0 .
$$

Using Lemma 4.7 and $a_{1} \alpha-h_{1}^{2}=3 c$, we have

$$
\left(e_{2} h_{1}\right)=2 c+\alpha a_{1}=5 c+h_{1}^{2} .
$$

By (13),

$$
-\left(c+h_{1}^{2}\right)+a_{1} g\left(\nabla_{\xi} e_{2}, e_{1}\right)+e_{2} h_{1}=0,
$$

from which we obtain

$$
a_{1} g\left(\nabla_{\xi} e_{2}, e_{1}\right)=-4 c,
$$

and so

$$
g\left(R\left(e_{1}, e_{2}\right) e_{2}, e_{1}\right)=-4 c .
$$

On the other hand, the equation of Gauss implies

$$
g\left(R\left(e_{1}, e_{2}\right) e_{2}, e_{1}\right)=4 c,
$$

and hence $c=0$. This is a contradiction.

Consequently, $M$ is a ruled real hypersurface.

From (2), any ruled real hypersurface satisfies $g\left(\left(\nabla_{X} S\right) Y, \xi\right)=0$ for any $X$ and $Y$ orthogonal to $\xi$, and $S \xi=\beta \xi$ for some function $\beta$.

From Theorems 3.1 and 4.1, we have Theorem 1.1.

\section{References}

[Berndt 1989] J. Berndt, "Real hypersurfaces with constant principal curvatures in complex hyperbolic space”, J. Reine Angew. Math. 395 (1989), 132-141. MR 90d:53062 Zbl 0655.53046

[Berndt and Brück 2001] J. Berndt and M. Brück, "Cohomogeneity one actions on hyperbolic spaces", J. Reine Angew. Math. 541 (2001), 209-235. MR 2002j:53059 Zbl 1014.53042

[Berndt and Tamaru 2007] J. Berndt and H. Tamaru, "Cohomogeneity one actions on noncompact symmetric spaces of rank one”, Trans. Amer. Math. Soc. 359:7 (2007), 3425-3438. MR 2008d:53063 Zbl 1117.53041

[Cecil and Ryan 1982] T. E. Cecil and P. J. Ryan, "Focal sets and real hypersurfaces in complex projective space”, Trans. Amer. Math. Soc. 269:2 (1982), 481-499. MR 83b:53049 Zbl 0492.53039

[Ki 1989] U.-H. Ki, "Real hypersurfaces with parallel Ricci tensor of a complex space form", Tsukuba J. Math. 13:1 (1989), 73-81. MR 90c:53135 Zbl 0678.53046 
[Ki et al. 1990] U.-H. Ki, H. Nakagawa, and Y. J. Suh, "Real hypersurfaces with harmonic Weyl tensor of a complex space form”, Hiroshima Math. J. 20:1 (1990), 93-102. MR 91c:53051 Zbl 0716.53026

[Kim and Ryan 2008] H. S. Kim and P. J. Ryan, "A classification of pseudo-Einstein hypersurfaces in $\mathbb{C P}^{2}$,, Differential Geom. Appl. 26:1 (2008), 106-112. MR 2008m:53135 Zbl 1143.53050

[Kimura 1986] M. Kimura, "Real hypersurfaces and complex submanifolds in complex projective space”, Trans. Amer. Math. Soc. 296:1 (1986), 137-149. MR 87k:53133 Zbl 0597.53021

[Kimura and Maeda 1989] M. Kimura and S. Maeda, "On real hypersurfaces of a complex projective space”, Math. Z. 202:3 (1989), 299-311. MR 90h:53067 Zbl 0661.53015

[Kon 1979] M. Kon, "Pseudo-Einstein real hypersurfaces in complex space forms", J. Differential Geom. 14:3 (1979), 339-354. MR 81k:53050 Zbl 0461.53031

[Kon 2014] M. Kon, "3-dimensional real hypersurfaces and Ricci operator", Differential Geom. Dyn. Syst. 16 (2014), 189-202. MR 3226614

[Lohnherr 1998] M. Lohnherr, On ruled real hypersurfaces of complex space forms, Ph.D. thesis, University of Cologne, 1998.

[Lohnherr and Reckziegel 1999] M. Lohnherr and H. Reckziegel, "On ruled real hypersurfaces in complex space forms", Geom. Dedicata 74:3 (1999), 267-286. MR 99m:53120 Zbl 0932.53018

[Maeda 1976] Y. Maeda, "On real hypersurfaces of a complex projective space”, J. Math. Soc. Japan 28:3 (1976), 529-540. MR 53 \#11543 Zbl 0324.53039

[Maeda 2013] S. Maeda, "Hopf hypersurfaces with $\eta$-parallel Ricci tensors in a nonflat complex space form”, Sci. Math. Jpn. 76:3 (2013), 449-456. MR 3310013

[Montiel 1985] S. Montiel, "Real hypersurfaces of a complex hyperbolic space", J. Math. Soc. Japan 37:3 (1985), 515-535. MR 86i:53027 Zbl 0554.53021

[Niebergall and Ryan 1997] R. Niebergall and P. J. Ryan, "Real hypersurfaces in complex space forms", pp. 233-305 in Tight and taut submanifolds (Berkeley, CA, 1994), edited by T. E. Cecil and S.-S. Chern, Math. Sci. Res. Inst. Publ. 32, Cambridge University Press, 1997. MR 98j:53066 Zbl 0904.53005

[Suh 1990] Y. J. Suh, "On real hypersurfaces of a complex space form with $\eta$-parallel Ricci tensor", Tsukuba J. Math. 14:1 (1990), 27-37. MR 91h:53047 Zbl 0721.53029

[Takagi 1973] R. Takagi, "On homogeneous real hypersurfaces in a complex projective space", Osaka J. Math. 10 (1973), 495-506. MR 49 \#1433 Zbl 0274.53062

[Takagi 1975a] R. Takagi, "Real hypersurfaces in a complex projective space with constant principal curvatures”, J. Math. Soc. Japan 27 (1975), 43-53. MR 50 \#8380 Zbl 0292.53042

[Takagi 1975b] R. Takagi, "Real hypersurfaces in a complex projective space with constant principal curvatures II”, J. Math. Soc. Japan 27:4 (1975), 507-516. MR 53 \#3955 Zbl 0311.53064

Received May 7, 2015. Revised July 21, 2015.

MAYUKO KON

FACULTY OF EDUCATION

SHINSHU UNIVERSITY

6-Ro, NishinAGANO

NAGANO CITY 380-8544

JAPAN

mayuko_k@shinshu-u.ac.jp 


\title{
PACIFIC JOURNAL OF MATHEMATICS
}

\author{
msp.org/pjm
}

Founded in 1951 by E. F. Beckenbach (1906-1982) and F. Wolf (1904-1989)

\section{EDITORS}

Don Blasius (Managing Editor)

Department of Mathematics

University of California

Los Angeles, CA 90095-1555

blasius@math.ucla.edu

\author{
Paul Balmer \\ Department of Mathematics \\ University of California \\ Los Angeles, CA 90095-1555 \\ balmer@math.ucla.edu \\ Robert Finn \\ Department of Mathematics \\ Stanford University \\ Stanford, CA 94305-2125 \\ finn@math.stanford.edu \\ Sorin Popa \\ Department of Mathematics \\ University of California \\ Los Angeles, CA 90095-1555 \\ popa@math.ucla.edu
}

\author{
Vyjayanthi Chari \\ Department of Mathematics \\ University of California \\ Riverside, CA 92521-0135 \\ chari@math.ucr.edu \\ Kefeng Liu \\ Department of Mathematics \\ University of California \\ Los Angeles, CA 90095-1555 \\ liu@math.ucla.edu \\ Jie Qing \\ Department of Mathematics \\ University of California \\ Santa Cruz, CA 95064 \\ qing@ cats.ucsc.edu
}

\section{PRODUCTION}

Silvio Levy, Scientific Editor, production@msp.org

\section{SUPPORTING INSTITUTIONS}

ACADEMIA SINICA, TAIPEI

CALIFORNIA INST. OF TECHNOLOGY

INST. DE MATEMÁTICA PURA E APLICADA

KEIO UNIVERSITY

MATH. SCIENCES RESEARCH INSTITUTE

NEW MEXICO STATE UNIV.

OREGON STATE UNIV.

\author{
STANFORD UNIVERSITY \\ UNIV. OF BRITISH COLUMBIA \\ UNIV. OF CALIFORNIA, BERKELEY \\ UNIV. OF CALIFORNIA, DAVIS \\ UNIV. OF CALIFORNIA, LOS ANGELES \\ UNIV. OF CALIFORNIA, RIVERSIDE \\ UNIV. OF CALIFORNIA, SAN DIEGO \\ UNIV. OF CALIF., SANTA BARBARA
}

\author{
Daryl Cooper \\ Department of Mathematics \\ University of California \\ Santa Barbara, CA 93106-3080 \\ cooper@math.ucsb.edu \\ Jiang-Hua Lu \\ Department of Mathematics \\ The University of Hong Kong \\ Pokfulam Rd., Hong Kong \\ jhlu@maths.hku.hk \\ Paul Yang \\ Department of Mathematics \\ Princeton University \\ Princeton NJ 08544-1000 \\ yang@math.princeton.edu
}

These supporting institutions contribute to the cost of publication of this Journal, but they are not owners or publishers and have no responsibility for its contents or policies.

See inside back cover or msp.org/pjm for submission instructions.

The subscription price for 2016 is US $\$ 440 /$ year for the electronic version, and $\$ 600 /$ year for print and electronic.

Subscriptions, requests for back issues and changes of subscribers address should be sent to Pacific Journal of Mathematics, P.O. Box 4163, Berkeley, CA 94704-0163, U.S.A. The Pacific Journal of Mathematics is indexed by Mathematical Reviews, Zentralblatt MATH, PASCAL CNRS Index, Referativnyi Zhurnal, Current Mathematical Publications and Web of Knowledge (Science Citation Index).

The Pacific Journal of Mathematics (ISSN 0030-8730) at the University of California, c/o Department of Mathematics, 798 Evans Hall \#3840, Berkeley, CA 94720-3840, is published twelve times a year. Periodical rate postage paid at Berkeley, CA 94704, and additional mailing offices. POSTMASTER: send address changes to Pacific Journal of Mathematics, P.O. Box 4163, Berkeley, CA 94704-0163.

PJM peer review and production are managed by EditFLOW ${ }^{\circledR}$ from Mathematical Sciences Publishers.

\section{PUBLISHED BY}

\section{mathematical sciences publishers \\ nonprofit scientific publishing}

http://msp.org/

(C) 2016 Mathematical Sciences Publishers 


\section{PACIFIC JOURNAL OF MATHEMATICS}

Volume $281 \quad$ No. $1 \quad$ March 2016

Compatible systems of symplectic Galois representations and the inverse Galois problem II: Transvections and huge image

SARA ARIAS-DE-REYNA, LUIS DIEULEFAIT and GABOR

WIESE

On the number of lines in the limit set for discrete subgroups of $\operatorname{PSL}(3, \mathbb{C})$

Waldemar Barrera, Angel CANo and JuÁn NaVArRete Galois theory, functional Lindemann-Weierstrass, and Manin maps

DANIEL BERTRAND and ANAND PILLAY

Morse area and Scharlemann-Thompson width for hyperbolic

3-manifolds

DiANE HOFFOSS and JOSEPH MAHER

Ricci tensor of real hypersurfaces

MAYUKO KON

Monotonicity formulae and vanishing theorems

JINTANG LI

Jet schemes of the closure of nilpotent orbits

ANNE MOREAU and RuPERT WeI TZE Yu

Components of spaces of curves with constrained curvature on flat surfaces

Nicolau C. Saldanha and Pedro ZüHlke A note on minimal graphs over certain unbounded domains of Hadamard manifolds

MiRIAM TELICHEVESKY 